\title{
Vilsmeier-Haack reagent: A facile synthesis of 2-(4-chloro-3,3-dimethyl-7- phenoxyindolin-2-ylidene)malonaldehyde and transformation into different heterocyclic compounds
}

\author{
Laya Roohi $^{a}$, Arash Afghan ${ }^{b^{*}}$ and Mehdi M. Baradarani ${ }^{a}$
}

${ }^{a}$ Department of Chemistry, Faculty of Science, University of Urmia, Urmia 57153-165, Iran

${ }^{b}$ Department of Chemical Engineering, Urmia University of Technology, Urmia 57155-419, Iran

CHRONICLE AB S T R A T

Article history:

Received March 20, 2013

Received in Revised form

July 7, 2013

Accepted 28 July 2013

Available online

30 July 2013

Keywords:

Vilsmeier-Haack reagent

Fischer indole synthesis

malonaldehydes

pyrazoles

enamines

cyanoacetamide

cyanopyridone
2-(5-Chloro-2-phenoxyphenyl)hydrazine was converted to corresponding $3 \mathrm{H}$-indole by Fischer method utilizing the isopropyl methyl ketone in acetic acid. The reaction of $3 \mathrm{H}$-indole with Vilsmeier-Haack reagent furnished aminomethylene malonaldehyde in excellent yield while the reactions of malonaldehyde with hydrazine, arylhydrazines, amines, cyanoacetamide and hydroxylamine hydrochloride, led to the corresponding pyrazole derivatives, enamines, cyanopyridone, and cyanoacetamide derivatives respectively.

\section{Introduction}

Chloromethyleneiminium salts, commonly known as highly versatile Vilsmeier-Haack reagent, ${ }^{1}$ usually generated in situ by the treatment of $\mathrm{POCl}_{3}$ with an $\mathrm{N}, \mathrm{N}$-disubstituted formamides (e.g., $\mathrm{DMF}$ ), is very useful in the synthetic transformations. Selected applications of this reagent include: formylation, ${ }^{2,3}$ cyclohaloaddition, ${ }^{4}$ cyclization $^{5}$ and ring annulations. ${ }^{6}$ A wide variety of alkene derivatives, ${ }^{7}$ carbonyl compounds, ${ }^{8}$ activated methyl and methylene groups bearing chemicals, ${ }^{9}$ and oxygen $^{10}$ as well as nitrogen nucleophiles ${ }^{11}$ undergo the reactions with Vilsmeier reagent to yield the corresponding iminium salts.

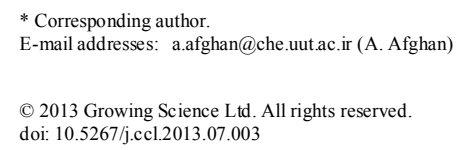


In 1959 , Fritz ${ }^{12}$ reported the $\mathrm{N}$-formylation of a 3,3-disubstituted $3 \mathrm{H}$-indole (indolenine) $\mathbf{1}$ leading to 2 by utilization of Vilsmeier reagent formed from DMF and $\mathrm{POCl}_{3}$. Further reaction of 2 with the Vilsmeier reagent, followed by hydrolysis produced compound 4. Formation of this product probably involves the intermediate $\mathbf{3}$, from which the $N$-formyl group is hydrolytically removed during workup (Scheme 1).
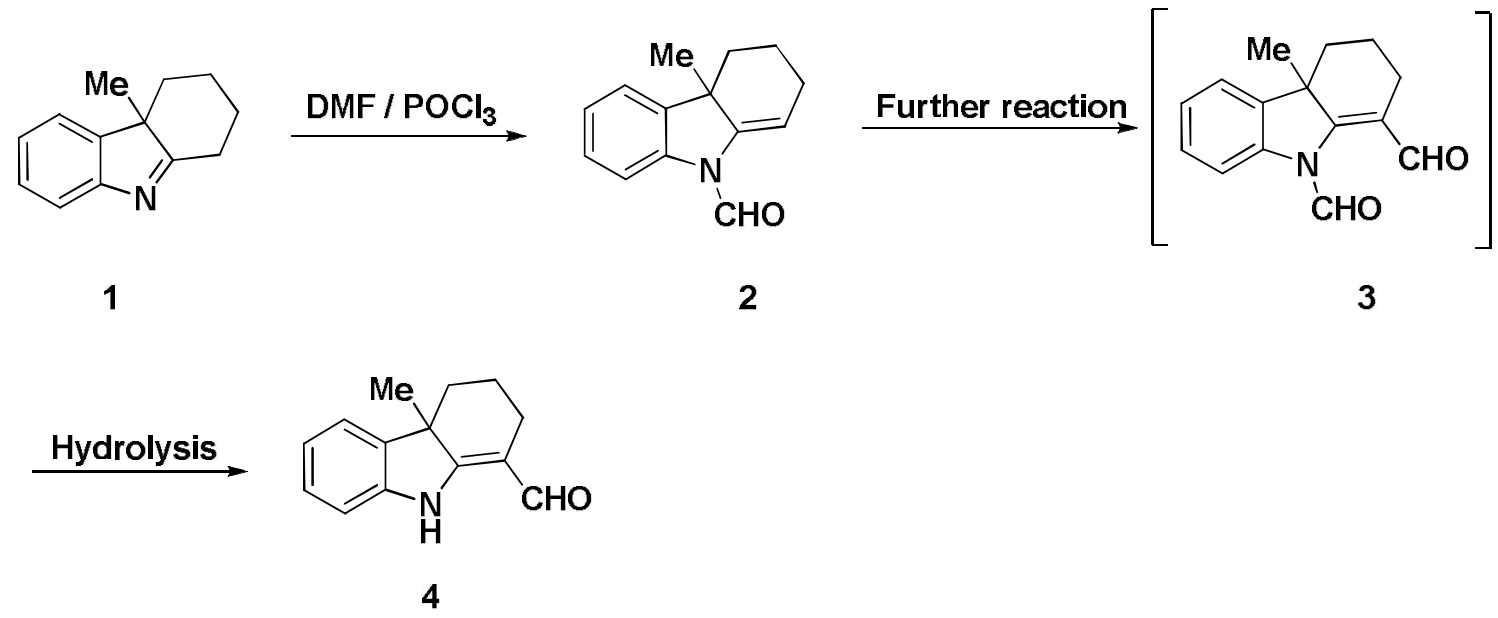

Scheme 1

Recently, we demonstrated ${ }^{13-15}$ that the 2- $\mathrm{CH}_{3}$ formylation reaction of some of 2,3,3trimethylindolenines ( $3 \mathrm{H}$-indoles) by Vilsmeier reagent furnished aminomethylene malonaldehydes. Thus formed 1,3-dialdehyde compounds undergo reaction with various nucleophiles to yield a wide range of new heterocyclic compounds. As an extension of our previous studies, herein we demonstrated the formylation of another indolenine to produce corresponding malonaldehyde as well as synthesis of various heterocyclic compounds by condensations of malonaldehyde with various arylhydrazines and cyanoacetamide leading to both 5- and 6-membered heterocycles, respectively.

\section{Results and Discussion}

5-Chloro-2-phenoxyaniline 5 was diazotized with $\mathrm{NaNO}_{2} / \mathrm{HCl}$. The formed diazonium salt was then reduced by stannous chloride dihydrate in $\mathrm{HCl}_{(\mathrm{aq})}$ to produce the corresponding bis- hydrazinium hexachloro stannate 6, revealed by atomic absorption analysis,. A neutralization of obtained reaction mixture by $\mathrm{NaOH}$ (aq.) furnished free base of aryl hydrazine 7 (Scheme 2).

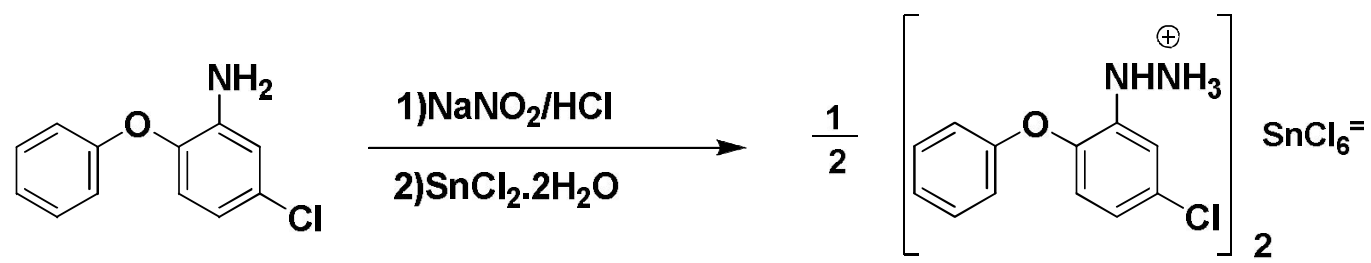

6

Scheme 2

Reaction of 7 with isopropyl methyl ketone in a Fischer reaction condition furnished the $3 H$ indole 8 in a good yield (Scheme 3). 
<smiles>CC(=O)C(C)C(C)C(=O)O</smiles>

\section{Scheme 3}

The structure of $3 H$-indole $\mathbf{8}$ was confirmed on the basis of analysis of ${ }^{1} \mathrm{H}-\mathrm{NMR}$ spectrum possessing six-hydrogen singlets for the geminal methyl groups, at $\delta 1.55 \mathrm{ppm}$ and three-hydrogen singlet signals for the imine-methyl group, at $\delta 2.29 \mathrm{ppm}$.

The reaction of 8 with Vilsmeier reagent at $75^{\circ} \mathrm{C}$, led to diformylation of imine-methyl group in excellent yield (Scheme 4). The structure of malonaldehyde 9 was confirmed by its spectral data. The IR absorptions at 3159 and $1675,1639 \mathrm{~cm}^{-1}$ support a presence of N-H and two carbonyl groups, thus in ${ }^{1} \mathrm{H}$-NMR spectrum signal for the $N$-hydrogen appearing at $\delta 13.55 \mathrm{ppm}$ and two aldehyde hydrogens at $\delta 9.75 \mathrm{ppm}$. The ${ }^{13} \mathrm{C}-\mathrm{NMR}$ spectrum of 9 showed the presence of two carbon signals at 187.66 and 192.64 ppm corresponding to $\mathrm{CHO}$ groups.<smiles>CC1=Nc2c(Cl)cccc2Oc2ccccc21</smiles>

8

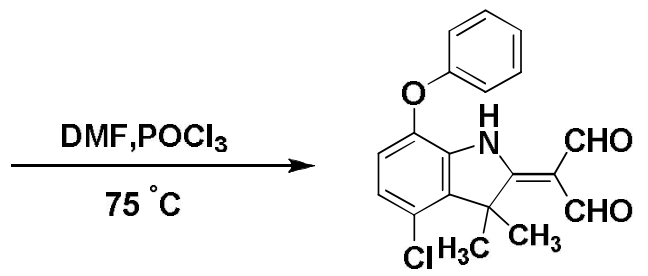

9

\section{Scheme 4}

1,3-Dicarbonyl compounds can be used as important building blocks in the syntheses of various heterocycles $^{16}$, which often show high biological activities ${ }^{16,17}$. The reaction of the substrate 9 with hydrazine hydrate and substituted arylhydrazines at room temperature and reflux conditions, respectively, afforded desired products 10a-d in quantitative yields (Scheme 5).

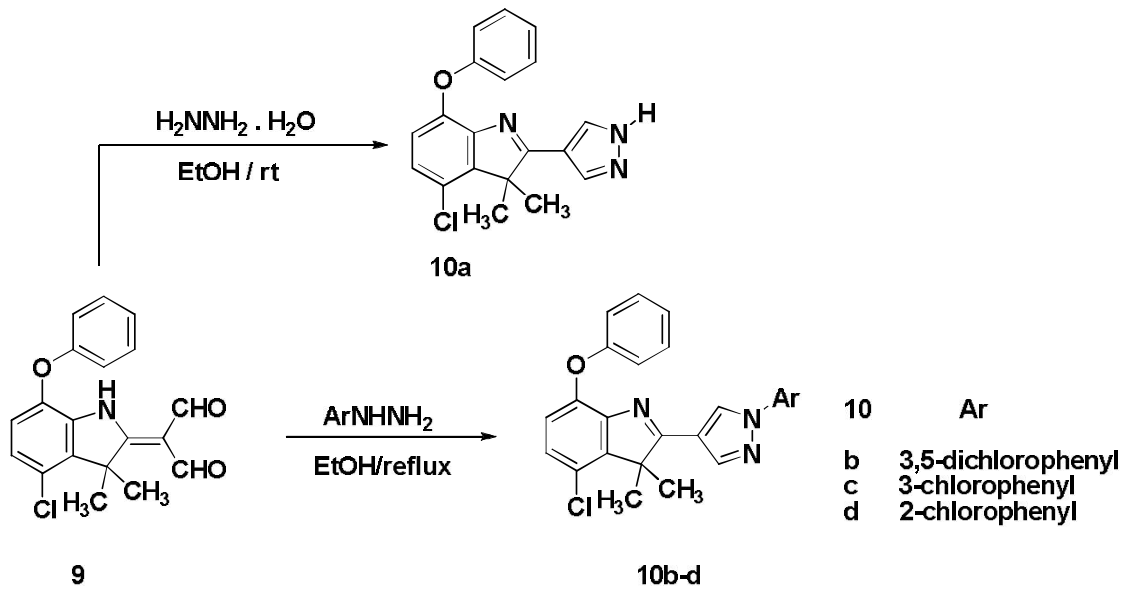

Scheme 5 
As extension of this work, we also examined the reactions of hydroxylamine hydrochloride, cyanoacetamide and arylamines with aminomethylene malonaldehyde 9.

The corresponding cyanoacetamide derivative $\mathbf{1 1}$ was readily achieved by refluxing a mixture of malonaldehyde 9 and hydroxylamine hydrochloride in ethanol (Scheme 6).<smiles>CC1(C)C(C=O)=C(C=O)Nc2c(Oc3ccccc3)ccc(Cl)c21</smiles>

9

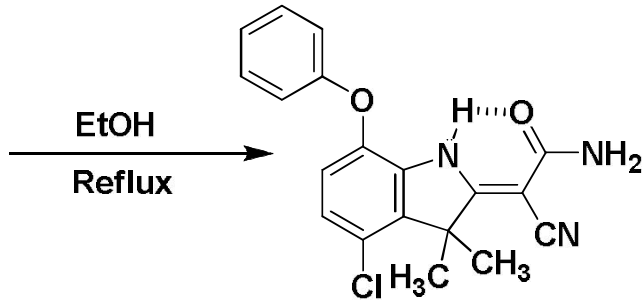

11

\section{Scheme 6}

The X-ray diffraction data for similar compound ${ }^{18}$ showed that the orientation of the acetamide group arises from intramolecular hydrogen bonding between the indole $\mathrm{N}-\mathrm{H}$ and the carbonyl group (Scheme 7).<smiles>Cc1ccc(Cl)c2c1C(C)(C)c1c(C#N)c(N)cn1-2</smiles>

Scheme 7

The compound 9 was allowed to react with cyanoacetamide under reflux condition in ethanol to obtain cyanopyridone derivative 12. The structure of compound $\mathbf{1 2}$ was elucidated from its spectral data. The ${ }^{1} \mathrm{H}-\mathrm{NMR}$ spectrum showed two singlet at $\delta 8.42$ and $8.66 \mathrm{ppm}$ respectively, belonged to two protons of pyridone. The broad singlet appearing around $\delta 13.07 \mathrm{ppm}$ confirmed the presence of pyridone N-H bond (Scheme 8).<smiles>CC1(C)C(=CC=O)Nc2c(Oc3ccccc3)ccc(Cl)c21</smiles>

9<smiles>CC1(C)C(c2c[nH]c(=O)c(C#N)c2)=Nc2c(Oc3ccccc3)ccc(Cl)c21</smiles>

12

Scheme 8 
Finally, attempt to synthesis quinoline derivatives starting from arylamines and malonaldehyde 9 was unsuccessful but this condensation gave enamines 13a-d in high yields (Scheme 9).

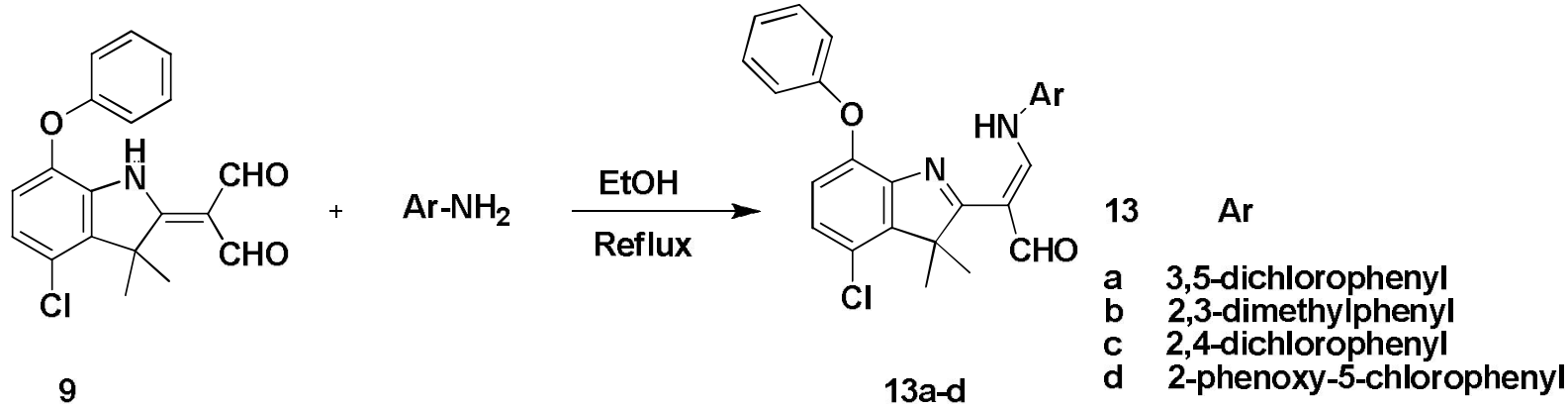

Scheme 9

A study of the ${ }^{1} \mathrm{H}-\mathrm{NMR}$ spectrum of enamine 13b showed that both $E$ and $Z$ isomers of $13 \mathrm{~b}$ have been formed in a ratio $1: 1$. Thus, there were two singlets at $\delta 8.39$ and $\delta 8.43 \mathrm{ppm}$ in a ratio $1: 1$, corresponding to $\mathrm{CH}=\mathrm{N}$ and a pair of siglets at $\delta 14.08$ and $\delta 14.12 \mathrm{ppm}$, again in a ratio of 1:1 assigned to NH protons (Scheme 10).<smiles>Cc1cccc(N/C=C(/C=O)C2=Nc3c(Oc4ccccc4)ccc(Cl)c3C2(C)C)c1C</smiles>

(E)<smiles>Cc1cccc(N/C=C(\C=O)C2=Nc3c(Oc4ccccc4)ccc(Cl)c3C2(C)C)c1C</smiles>

(Z)

Scheme 10

\section{Conclusion}

In summary, we have examined the reaction of $3 H$-indole with Vilsmeier-Haack reagent. This study revealed that the formed malonaldehyde from formylation reaction could be cyclized using various arylhydrazines and cyanoacetamide to afford substituted pyrazoles and cyanopyridone. Also, reaction of malonaldehyde with hydroxylamine and primary aromatic amines furnished cyanoacetamide and enamine derivatives, respectively.

\section{Acknowledgements}

The authors are grateful to the University of Urmia for financial support of this work.

\section{Experimental}

Melting points were measured on an electrothermal IA9200 apparatus. ${ }^{1} \mathrm{H}$ NMR and ${ }^{13} \mathrm{C}$ NMR spectra were recorded on a Bruker Avance AQS $300 \mathrm{MHz}$ spectrometer, at $300 \mathrm{MHz}$ and $75 \mathrm{MHz}$ 
respectively. Chemical shifts $\delta$ are in parts per million (ppm) measured in $\mathrm{CDCl}_{3}$ and DMSO- $d_{6}$ as solvents and related to TMS as the internal standard. IR spectra were recorded on a ThermonicoletNexus 670 FT-IR instrument. Elemental analyses were performed on Heraeus CHN-O rapid analyzer.

\section{4-Chloro-2,3,3-trimethyl-7-phenoxy-3H-indole (8).}

A mixture of arylhydrazine 7 (5 g, $21 \mathrm{mmol})$ and isopropyl methyl ketone $(2.01 \mathrm{~g}, 23 \mathrm{mmol})$ was heated at reflux in acetic acid $(20 \mathrm{~mL})$ overnight and then cooled, diluted with $\mathrm{H}_{2} \mathrm{O}(50 \mathrm{~mL})$, and neutralized with $\mathrm{NaOH}$ solution $(2 \mathrm{~N})$, the aqueous solution was extracted with EtOAc $(3 \times 30 \mathrm{~mL})$. The combined organic layers dried over $\mathrm{Na}_{2} \mathrm{SO}_{4}$ and solvent was evaporated to give $14(4 \mathrm{~g}, 65 \%)$ as a viscous oil which was solidified on standing; FT-IR (KBr) $v_{\max } / \mathrm{cm}^{-1}: 3065,2969,2930,2869,1592$, 1261, 752; ${ }^{1} \mathrm{H}$ NMR $\left(\mathrm{CDCl}_{3}\right): \delta(\mathrm{ppm}) 1.55\left(\mathrm{~s}, 6 \mathrm{H}, 2 \times \mathrm{CH}_{3}\right), 2.29\left(\mathrm{~s}, 3 \mathrm{H}, \times \mathrm{CH}_{3}\right), 6.74(\mathrm{~d}, J=8.7 \mathrm{~Hz}$, $1 \mathrm{H}), 7.01(\mathrm{~d}, J=8.7 \mathrm{~Hz}, 1 \mathrm{H}), 7.07-7.13(\mathrm{~m}, 3 \mathrm{H}), 7.36(\mathrm{t}, J=8 \mathrm{~Hz}, 2 \mathrm{H}) ;{ }^{13} \mathrm{C} \mathrm{NMR}\left(\mathrm{CDCl}_{3}\right): \delta(\mathrm{ppm})$ 14.36, 20.44, 55.47, 116.75, 117.37, 118.66, 122.97, 125.67, 129.05, 142.25, 146.28, 155.70, 187.72; Anal. Calcd. for $\mathrm{C}_{17} \mathrm{H}_{16} \mathrm{ClNO}: \mathrm{C}, 71.45 \%$; H, 5.64\%;N, 4.90\%. Found: $\mathrm{C}, 71.38 ; \mathrm{H}, 5.40 \%$; N, $4.82 \%$.

\section{2-(4-Chloro-3,3-dimethyl-7-phenoxyindolin-2-ylidene)malonaldehyde (9).}

To $N, N$-dimethyl- formamide $(3.5 \mathrm{~mL}, 45.6 \mathrm{mmol})$ cooled in an ice bath was added dropwise phosphorus oxychloride $(2.08 \mathrm{~mL}, 22.8 \mathrm{mmol})$ with stirring at below $5{ }^{\circ} \mathrm{C}$. After that addition, $3 \mathrm{H}-$ indole 8 (2.18 $\mathrm{g}, 7.6 \mathrm{mmol})$ was added slowly. The cooling bath was removed and the reaction mixture was stirred at $75{ }^{\circ} \mathrm{C}$ for $6 \mathrm{~h}$. The resulting solution was added to ice-cooled water and made alkaline with $\mathrm{NaOH}$ (aq.) solution $(\mathrm{pH}=8-9)$. The resulting precipitate was collected by filtration, dried in air and recrystallized from ethanol to give yellow crystals. Yield $84 \%$; mp $178-180{ }^{\circ} \mathrm{C}$; FTIR (KBr) $v_{\max } / \mathrm{cm}^{-1}: 3159,3067,2978,2937,2733,1675,1639,1259,767 ;{ }^{1} \mathrm{H} \mathrm{NMR}\left(\mathrm{CDCl}_{3}\right): \delta$ (ppm) $1.95\left(\mathrm{~s}, 6 \mathrm{H}, 2 \times \mathrm{CH}_{3}\right), 6.79(\mathrm{~d}, J=8.7 \mathrm{~Hz}, 1 \mathrm{H}), 7.05(\mathrm{~d}, J=8.7 \mathrm{~Hz}, 1 \mathrm{H}), 7.09(\mathrm{~d}, J=7.8,2 \mathrm{H}), 7.21$ $(\mathrm{t}, J=7.2 \mathrm{~Hz}, 1 \mathrm{H}), 7.41(\mathrm{t}, J=7.5 \mathrm{~Hz}, 2 \mathrm{H}), 9.79(\mathrm{~s}, 2 \mathrm{H}, 2 \times \mathrm{CHO}), 13.55(\mathrm{bs}, 1 \mathrm{H},-\mathrm{NH}) ;{ }^{13} \mathrm{C}$ NMR $\left(\mathrm{CDCl}_{3}\right): \delta(\mathrm{ppm}) 19.69,53.86,109.23,118.37,119.11,123.53,124.76,127.32,130.20,131.69$, 137.43, 142.36, 155.31, 178.50, 187.66, 192.64; Anal. Calcd. for $\mathrm{C}_{19} \mathrm{H}_{16} \mathrm{ClNO}_{3}: \mathrm{C}, 66.77 \%$; $\mathrm{H}$, $4.72 \% ; \mathrm{N}, 4.10 \%$. Found: $\mathrm{C}, 66.70 ; \mathrm{H}, 7.85 \% ; \mathrm{N}, 4.22 \%$.

\section{4-Chloro-3,3-dimethyl-7-phenoxy-2-(1H-pyrazol-4-yl)-3H-indole (10a).}

A mixture of the malonaldehyde $9(0.1 \mathrm{~g}, 0.29 \mathrm{mmol})$ and hydrazine monohydrate $(0.09 \mathrm{~g}, 1.46$ $\mathrm{mmol})$, in absolute ethanol $(15 \mathrm{~mL})$ was stirred at room temperature overnight. After this time, the solvent evaporated and ethyl acetate $(20 \mathrm{~mL})$ was added to the residue. The organic layer was washed with water, dried over $\mathrm{Na}_{2} \mathrm{SO}_{4}$ and solvent evaporated. Resulting yellow precipitate crystallized from ethanol. Yield $92 \%$; $\mathrm{mp} 211-213^{\circ} \mathrm{C}$; FT-IR $(\mathrm{KBr}) v_{\max } / \mathrm{cm}^{-1}: 3160,2973,2934,1593,1569,1472$, 1259, 954, 778; ${ }^{1} \mathrm{H}$ NMR $\left(\mathrm{CDCl}_{3}\right): \delta(\mathrm{ppm}) 1.63\left(\mathrm{~s}, 6 \mathrm{H}, 2 \times \mathrm{CH}_{3}\right), 6.74(\mathrm{~d}, J=8.4 \mathrm{~Hz}, 1 \mathrm{H}), 7.01-7.11$ $(\mathrm{m}, 4 \mathrm{H}), 7.26-7.32(\mathrm{~m}, 2 \mathrm{H}), 8.37\left(\mathrm{~s}, 2 \mathrm{H}\right.$, Pyrazole), $10.22(\mathrm{bs}, 1 \mathrm{H}, \mathrm{NH}) ;{ }^{13} \mathrm{C} \mathrm{NMR}\left(\mathrm{CDCl}_{3}\right): \delta(\mathrm{ppm})$ 21.38, 55.95, 117.00, 118.32, 119.45, 122.95, 123.97, 126.92, 129.87, 135.25, 143.19, 144.38, 147.42, 156.39, 179.97; Anal. Calcd. for $\mathrm{C}_{19} \mathrm{H}_{16} \mathrm{ClN}_{3} \mathrm{O}$ : C, 67.56\%; H, 4.77\%; $\mathrm{N}, 12.44 \%$. Found: $\mathrm{C}$, $67.70 ; \mathrm{H}, 4.88 \% ; \mathrm{N}, 12.32 \%$.

\section{General procedure for synthesis of (10b-d).}

A mixture of the malonaldehyde $9(0.1 \mathrm{~g}, 0.29 \mathrm{mmol})$ and aryl hydrazinium chloride $(0.29 \mathrm{mmol})$, in absolute ethanol $(15 \mathrm{~mL})$ was heated at reflux and stirred for 2-5 $\mathrm{h}$. After cooling and concentrating the resulting crystals were collected by filtration and recrystallized from ethanol to give the corresponding pyrazoles. 
4-Chloro-2-(1-(3,5-dichlorophenyl)-1H-pyrazol-4-yl)-3,3-dimethyl-7-phenoxy-3H-indole (10b).

Yield 71\%; mp 165-168 ${ }^{\circ} \mathrm{C}$; FT-IR (KBr) $v_{\max } / \mathrm{cm}^{-1}: 3119,3087,2974,2933,1591,1467,1258,779$; ${ }^{1} \mathrm{H}$ NMR $\left(\mathrm{CDCl}_{3}\right): \delta(\mathrm{ppm}) 1.79\left(\mathrm{~s}, 6 \mathrm{H}, 2 \times \mathrm{CH}_{3}\right), 6.78(\mathrm{~d}, J=7.8 \mathrm{~Hz}, 1 \mathrm{H}), 7.06(\mathrm{~d}, J=7.8 \mathrm{~Hz}, 1 \mathrm{H})$, 7.13-7.15 (m, 3H), 7.33-7.38 (m, 3H), $7.71(\mathrm{~s}, 2 \mathrm{H}), 8.33$ (s, 1H, Pyrazole), $8.68\left(\mathrm{~s}, 1 \mathrm{H}\right.$, Pyrazole) ${ }^{13} \mathrm{C}$ NMR $\left(\mathrm{CDCl}_{3}\right): \delta(\mathrm{ppm}) 21.16,55.59,117.80,118.22,118.54,119.56,122.90,123.89,127.16$, $127.87,129.79$, 136.11, 140.74, 141.54, 143.42, 148.00, 156.62, 177.95; Anal. Calcd. for $\mathrm{C}_{25} \mathrm{H}_{18} \mathrm{C}_{13} \mathrm{~N}_{3} \mathrm{O}$ : C, $62.19 \%$; H, 3.76\%; N, 8.70\%. Found: C, 62.26; H, 3.88\%; N, 8.55\%.

\section{4-Chloro-2-(1-(3-chlorophenyl)-1H-pyrazol-4-yl)-3,3-dimethyl-7-phenoxy-3H-indole (10c).}

Yield 77\%; mp 143-145 ${ }^{\circ} \mathrm{C}$; FT-IR (KBr) $v_{\max } / \mathrm{cm}^{-1}: 3114,2969,2929,2870,1594,1483,1203,956$, $771 ;{ }^{1} \mathrm{H} \mathrm{NMR}\left(\mathrm{CDCl}_{3}\right): \delta(\mathrm{ppm}) \delta(\mathrm{ppm}) 1.76\left(\mathrm{~s}, 6 \mathrm{H}, 2 \times \mathrm{CH}_{3}\right), 6.78(\mathrm{~d}, J=8.7 \mathrm{~Hz}, 1 \mathrm{H}), 7.06(\mathrm{~d}, J=8.7$ $\mathrm{Hz}, 1 \mathrm{H}), 7.15(\mathrm{~d}, J=7.5,2 \mathrm{H}), 7.16(\mathrm{t}, J=7.5 \mathrm{~Hz}, 1 \mathrm{H}), 7.32(\mathrm{~d}, J=8.1 \mathrm{~Hz}, 1 \mathrm{H}), 7.38(\mathrm{t}, J=8.7,1 \mathrm{H}), 7.42$ $(\mathrm{t}, J=7.2,2 \mathrm{H}), 7.65(\mathrm{~d}, J=8.1,1 \mathrm{H}), 7.84(\mathrm{~s}, 1 \mathrm{H}), 8.33(\mathrm{~s}, 1 \mathrm{H}$, Pyrazole $), 8.73(\mathrm{~s}, 1 \mathrm{H}$, Pyrazole $) ;{ }^{13} \mathrm{C}$ NMR $\left(\mathrm{CDCl}_{3}\right): \delta(\mathrm{ppm}) 21.26,55.92,117.27,117.53,118.53,119.56,119.82,122.89,123.90$, $127.18,127.43,128.33,129.79,130.68,135.48,140.24,141.21,143.25,147.84,156.58,178.32$; Anal. Calcd. for $\mathrm{C}_{25} \mathrm{H}_{19} \mathrm{Cl}_{2} \mathrm{~N}_{3} \mathrm{O}: \mathrm{C}, 66.97 \% ; \mathrm{H}, 4.27 \% ; \mathrm{N}, 9.37 \%$. Found: $\mathrm{C}, 66.82 ; \mathrm{H}, 4.38 \%$; , $9.55 \%$.

\section{4-Chloro-2-(1-(2-chlorophenyl)-1H-pyrazol-4-yl)-3,3-dimethyl-7-phenoxy-3H-indole (10d).}

Yield 62\%; mp 123-125 ${ }^{\circ} \mathrm{C}$; FT-IR (KBr) $v_{\max } / \mathrm{cm}^{-1}: 3125,3068,2974,2932,2875,1567,1487$, 1248, 954, 753; ${ }^{1} \mathrm{H}$ NMR $\left(\mathrm{CDCl}_{3}\right): \delta(\mathrm{ppm}) 1.74\left(\mathrm{~s}, 6 \mathrm{H}, 2 \times \mathrm{CH}_{3}\right), 6.78(\mathrm{~d}, J=8.7 \mathrm{~Hz}, 1 \mathrm{H}), 7.04(\mathrm{~d}$, $J=8.7 \mathrm{~Hz}, 1 \mathrm{H}), 7.12-7.15(\mathrm{~m}, 3 \mathrm{H}), 7.34-7.43(\mathrm{~m}, 4 \mathrm{H}), 7.55\left(\mathrm{dd}, J_{o}=7.5 \mathrm{~Hz}, \mathrm{~J}_{\mathrm{m}}=2 \mathrm{~Hz}, 1 \mathrm{H}\right), 7.63$ (dd, $\left.J_{o}=7.5 \mathrm{~Hz}, \mathrm{~J}_{\mathrm{m}}=2 \mathrm{~Hz}, 1 \mathrm{H}\right), 8.39(\mathrm{~s}, 1 \mathrm{H}$, Pyrazole $), 8.54(\mathrm{~s}, 1 \mathrm{H}$, Pyrazole $) ;{ }^{13} \mathrm{C} \mathrm{NMR}\left(\mathrm{CDCl}_{3}\right): \delta(\mathrm{ppm})$ 20.26, 54.96, 115.77, 117.64, 118.41, 121.93, 122.67, 125.78, 126.63, 126.82, 127.28, 128.66, $128.70,129,78,131.10,136.48,140.00,142.65,144.65,146.84,155.82,177.44$; Anal. Calcd. for $\mathrm{C}_{25} \mathrm{H}_{19} \mathrm{Cl}_{2} \mathrm{~N}_{3} \mathrm{O}$ : C, 66.97\%; H, 4.27\%; N, 9.37\%. Found: C, 66.82; H, 4.38\%; N, 9.55\%.

\section{(Z)-2-(4-Chloro-3,3-dimethyl-7-phenoxyindolin-2-ylidene)-2-cyanoacetamide (11).}

A solution of malonaldehyde $9(0.1 \mathrm{~g}, 0.29 \mathrm{mmol})$ and hydroxylamine hydrochloride $(0.02 \mathrm{~g}, 0.29$ mmol) in absolute ethanol $(15 \mathrm{~mL})$ was refluxed for $2 \mathrm{hr}$. After this time, a solution standing overnight and then yellow precipitate was filtered off, washed with water and air- dried product was purified by recrystallization from ethanol. Yield 78\%; mp $123-125^{\circ} \mathrm{C}$; FT-IR $(\mathrm{KBr}) v_{\mathrm{max}} / \mathrm{cm}^{-1}: 3477$, $3318,3169,2983,2941,2189,1658,1554,1225,780 ;{ }^{1} \mathrm{H} \mathrm{NMR}\left(\mathrm{CDCl}_{3}\right): \delta(\mathrm{ppm}) 1.88(\mathrm{~s}, 6 \mathrm{H}$, $\left.2 \times \mathrm{CH}_{3}\right), 5.40\left(\mathrm{bs}, \mathrm{H}, \mathrm{NH}_{2}\right), 5.95\left(\mathrm{~s}, 1 \mathrm{H}, \mathrm{NH}_{2}\right), 6.77(\mathrm{~d}, J=8.4 \mathrm{~Hz}, 1 \mathrm{H}), 6.93(\mathrm{~d}, J=8.4 \mathrm{~Hz}, 1 \mathrm{H}), 7.04$ $(\mathrm{d}, J=7.2 \mathrm{~Hz}, 2 \mathrm{H}), 7.17(\mathrm{t}, J=6.3 \mathrm{~Hz}, 1 \mathrm{H}), 7.38(\mathrm{t}, J=7.2 \mathrm{~Hz}, 2 \mathrm{H}), 11.71(\mathrm{bs}, 1 \mathrm{H}, \mathrm{NH}) ;{ }^{13} \mathrm{C} \mathrm{NMR}$ $\left(\mathrm{CDCl}_{3}\right): \delta(\mathrm{ppm}) 21.03,52.00,69.85,118.64,118.80,119.06,123.91,124.36,125.34,130.12$, 133.10, 134.72, 140.68, 155.72, 168.89, 176.59; Anal. Calcd. for $\mathrm{C}_{19} \mathrm{H}_{16} \mathrm{ClN}_{3} \mathrm{O}_{2}: \mathrm{C}, 64.50 \%$; $\mathrm{H}$, 4.56\%;N, 11.88\%. Found: C, 64.76; H, 4.38\%; N, 11.55\%.

5-(4-Chloro-3,3-dimethyl-7-phenoxy-3H-indol-2-yl)-2-oxo-1,2-dihydropyridine-3-carbo- nitrile (12).

Cyanoacetamide $(0.04 \mathrm{~g}, 0.48 \mathrm{mmol})$ was dissolved in hot EtOH $(10 \mathrm{~mL}, 95 \%)$, then piperidine $(0.11$ g, $0.12 \mathrm{~mL}, 1.25 \mathrm{mmol}$ ) was added and the mixture was heated for $10 \mathrm{~min}$. The malonaldehyde 9 $(0.15 \mathrm{~g}, 0.44 \mathrm{mmol})$ was slowly added and the reaction mixture was refluxed overnight. After cooling the solution, formed precipitate was filtered off, washed with aqueous ethanol and finally dried in air. Yield 82\%; mp 269-270 ${ }^{\circ} \mathrm{C}$; FT-IR (KBr) $v_{\max } / \mathrm{cm}^{-1}: 3158,3070,2980,2923,2230,1656,1248,753$; ${ }^{1} \mathrm{H} \mathrm{NMR}\left(\mathrm{CDCl}_{3}\right): \delta(\mathrm{ppm}) 1.63\left(\mathrm{~s}, 6 \mathrm{H}, 2 \times \mathrm{CH}_{3}\right), 6.91(\mathrm{~d}, J=8.4 \mathrm{~Hz}, 1 \mathrm{H}), 7.03(\mathrm{~d}, J=7.2 \mathrm{~Hz}, 2 \mathrm{H}), 7.13$ (t, $J=6.9,1 \mathrm{H}), 7.22(\mathrm{~d}, J=8.4,1 \mathrm{H}), 7.31(\mathrm{t}, J=6.9,2 \mathrm{H}), 8.42(\mathrm{~s}, 1 \mathrm{H}$, pyridine $), 8.66$ (, $1 \mathrm{H}$, pyridine), 13.07 (bs, $1 \mathrm{H},-\mathrm{NH}) ;{ }^{13} \mathrm{C} \mathrm{NMR}\left(\mathrm{CDCl}_{3}\right)$ : $\delta(\mathrm{ppm})$ 20.46, 55.66, 104.84, 111.04, 116.03, 118.63, 
$120.51,122.80,123.89,128.10,130.44,143.07,144.68,144.99,147.25,147.75,157.30,159.84$, 178.85; Anal. Calcd. for $\mathrm{C}_{22} \mathrm{H}_{16} \mathrm{ClN}_{3} \mathrm{O}_{2}$ : C, 67.78\%; H, 4.14\%;, $10.78 \%$. Found: $\mathrm{C}, 67.76 ; \mathrm{H}$, $4.28 \% ; \mathrm{N}, 10.55 \%$.

\section{General procedure for synthesis of (13a-d).}

Primary aromatic amines $(0.29 \mathrm{mmol})$ was added to the solution of polyphosphoric acid $(0.06 \mathrm{~g})$ in absolute ethanol $(15 \mathrm{~mL})$ at $70{ }^{\circ} \mathrm{C}$. After 10 minutes, malonaldehyde $9(0.29 \mathrm{mmol})$ was added to the hot solution and reaction mixture was refluxed overnight. After cooling the reaction mixture, resulting precipitate was collected, washed with ethanol and dried in air. The crude products recrystallized from ethanol.

(E)-2-(4-Chloro-3,3-dimethyl-7-phenoxy-3H-indol-2-yl)-3-(3,5-dichlorophenylamino)acrylaldehyde (13a).

Yield 78\%; mp 158-159 ${ }^{\circ} \mathrm{C}$; FT-IR (KBr) $v_{\max } / \mathrm{cm}^{-1}: 3065,2928,1665,1629,1487,1250,776 ;{ }^{1} \mathrm{H}$ $\operatorname{NMR}\left(\mathrm{CDCl}_{3}\right): \delta(\mathrm{ppm}) 1.86\left(\mathrm{~s}, 6 \mathrm{H}, 2 \times \mathrm{CH}_{3}\right), 6.69(\mathrm{~s}, 2 \mathrm{H}), 7.00-7.17(\mathrm{~m}, 6 \mathrm{H}), 7.35(\mathrm{t}, J=6.9 \mathrm{~Hz}, 1 \mathrm{H})$, $8.24(\mathrm{~s}, 1 \mathrm{H},-\mathrm{CHO}), 12-15$ (bs, $1 \mathrm{H}, \mathrm{NH}) ;{ }^{13} \mathrm{C} \mathrm{NMR}\left(\mathrm{CDCl}_{3}\right): \delta(\mathrm{ppm}) 19.78,56.40,108.22,115.54$, $116.65,118.37,119.07,122.09,122.55,124.88,125.38,127.43,130.10,135.99,142.12,143.10$, 153.25, 157.44, 182.72, 186.79. Anal. Calcd. for $\mathrm{C}_{25} \mathrm{H}_{19} \mathrm{Cl}_{3} \mathrm{~N}_{2} \mathrm{O}_{2}$ : C, 61.81\%; H, 3.94\%; N, 5.77\%. Found: C, 61.68; H, 3.85\%; N, 5.65\%.

\section{2-(4-Chloro-3,3-dimethyl-7-phenoxy-3H-indol-2-yl)-3-(2,3-dimethylphenylamino)acryl aldehyde (13b).}

Yield 92\%; mp 115-117 ${ }^{\circ} \mathrm{C}$; FT-IR $(\mathrm{KBr}) v_{\max } / \mathrm{cm}^{-1}: 3065,2971,2930,2870,2724,1664,1619$, 1486, 1253, 770; ${ }^{1} \mathrm{H}$ NMR $\left(\mathrm{CDCl}_{3}\right): \delta(\mathrm{ppm}) 1.88(\mathrm{~s}, 6 \mathrm{H}, 2 \times \mathrm{CH} 3), 2.24\left(\mathrm{~s}, 3 \mathrm{H}, \mathrm{CH}_{3}\right), 2,32(\mathrm{~s}, 3 \mathrm{H}$, $\left.\mathrm{CH}_{3}\right), 6.79(\mathrm{~d}, \mathrm{~J}=7.8 \mathrm{~Hz}, 1 \mathrm{H}), 7.019-7.069(\mathrm{~m}, 4 \mathrm{H}), 7.10(\mathrm{~d}, J=7.8 \mathrm{~Hz}, 2 \mathrm{H}), 7.16(\mathrm{~d}, J=6 \mathrm{~Hz}, 2 \mathrm{H})$, $7.34(\mathrm{t}, J=7.8 \mathrm{~Hz}, 2 \mathrm{H}), 8.39(\mathrm{~s}, 1 \mathrm{H}, \mathrm{H}-\mathrm{C}=\mathrm{N}$, One isomer), $8.43(\mathrm{~s}, 1 \mathrm{H}, \mathrm{H}-\mathrm{C}=\mathrm{N}$, Another isomer) (9.73 (s, 1H, -CHO), 14.08 (bs, 1H, -NH, One isomer), 14.12 (bs, $1 \mathrm{H},-\mathrm{NH}$, Another isomer); ${ }^{13} \mathrm{C}$ NMR $\left(\mathrm{CDCl}_{3}\right): \delta(\mathrm{ppm}) 12.39,18.62,19.47,55.65,106.65,113.31,117.34,117.96,122.28,125.43$, 125.56, 126.52, 128.74, 137.24, 137.30, 141.73, 143.03, 144.83, 153.00, 155.99, 182.76, 185.96. Anal. Calcd. for $\mathrm{C}_{27} \mathrm{H}_{25} \mathrm{ClN}_{2} \mathrm{O}_{2}$ : C, 72.88\%; H, 5.66\%;N, 6.30\%. Found: C, 72.68; H, 5.81\%; N, $6.35 \%$.

\section{3-(5-Chloro-2-phenoxyphenylamino)-2-(4-chloro-3,3-dimethyl-7-phenoxy-3H-indol-2-yl) acrylaldehyde (13c).}

Yield 86\%; mp 138-139 ${ }^{\circ} \mathrm{C}$; FT-IR (KBr) $v_{\max } / \mathrm{cm}^{-1}:$ 3066, 2976, 2936, 2871, 2734, 1678, 1617, 1494, 1255, 749; ${ }^{1} \mathrm{H}$ NMR $\left(\mathrm{CDCl}_{3}\right): \delta(\mathrm{ppm}) 1.82\left(\mathrm{~s}, 6 \mathrm{H}, 2 \times \mathrm{CH}_{3}\right), 6.56(\mathrm{~d}, J=8.7,1 \mathrm{H}), 6.65(\mathrm{~d}, J=$ $8.7,1 \mathrm{H}), 6.81(\mathrm{~d}, J=7.9,2 \mathrm{H}), 6.86(\mathrm{~d}, J=7.9,2 \mathrm{H}), 6.93(\mathrm{~d}, J=7.9,1 \mathrm{H}), 6.97-7.00(\mathrm{~m}, 2 \mathrm{H}), 7.04-$ $7.11(\mathrm{~m}, 3 \mathrm{H}), 7.21(\mathrm{t}, J=6.5 \mathrm{~Hz}, 2 \mathrm{H}), 7.29(\mathrm{~d}, J=2.2,1 \mathrm{H}), 8.40(\mathrm{~s}, 1 \mathrm{H}, \mathrm{CH}=\mathrm{N}), 9.74(\mathrm{~s}, 1 \mathrm{H},-\mathrm{CHO})$, $13.94(\mathrm{bs}, 1 \mathrm{H},-\mathrm{NH}) ;{ }^{13} \mathrm{C} \mathrm{NMR}\left(\mathrm{CDCl}_{3}\right): \delta(\mathrm{ppm}) 18.52,55.38,107.62,116.56,116.65,117.61$, $118.69,118.96,121.43,122.63,123.41,124.48,125.66,127.42,128.66,128.73,130.81,141.38$, $141.45,146.11,146.46,152.20,154.24,155.24,181.65,186.18$. Anal. Calcd. for $\mathrm{C}_{31} \mathrm{H}_{24} \mathrm{Cl}_{2} \mathrm{~N}_{2} \mathrm{O}_{3}: \mathrm{C}$, $68.51 \%$; H, 4.45\%;N, 5.15\%. Found: C, 68.86; H, 4.70\%; N, 5.42\%.

\section{3-(5-Chloro-2-phenoxyphenylamino)-2-(4-chloro-3,3-dimethyl-7-phenoxy-3H-indol-2-yl) acrylaldehyde (13d).}

Yield 86\%; mp 138-139 ${ }^{\circ} \mathrm{C}$; FT-IR (KBr) $v_{\max } / \mathrm{cm}^{-1}$ : 3066, 2976, 2936, 2871, 2734, 1678, 1617 , 1494, 1255, 749; ${ }^{1} \mathrm{H}$ NMR $\left(\mathrm{CDCl}_{3}\right): \delta(\mathrm{ppm}) 1.82\left(\mathrm{~s}, 6 \mathrm{H}, 2 \times \mathrm{CH}_{3}\right), 6.56(\mathrm{~d}, J=8.7,1 \mathrm{H}), 6.65(\mathrm{~d}, J=$ $8.7,1 \mathrm{H}), 6.81(\mathrm{~d}, J=7.9,2 \mathrm{H}), 6.86(\mathrm{~d}, J=7.9,2 \mathrm{H}), 6.93(\mathrm{~d}, J=7.9,1 \mathrm{H}), 6.97-7.00(\mathrm{~m}, 2 \mathrm{H}), 7.04-$ 
$7.11(\mathrm{~m}, 3 \mathrm{H}), 7.21(\mathrm{t}, J=6.5 \mathrm{~Hz}, 2 \mathrm{H}), 7.29(\mathrm{~d}, J=2.2,1 \mathrm{H}), 8.40(\mathrm{~s}, 1 \mathrm{H}, \mathrm{CH}=\mathrm{N}), 9.74(\mathrm{~s}, 1 \mathrm{H},-\mathrm{CHO})$, $13.94(\mathrm{bs}, 1 \mathrm{H},-\mathrm{NH}) ;{ }^{13} \mathrm{C}$ NMR $\left(\mathrm{CDCl}_{3}\right): \delta(\mathrm{ppm}) 18.52,55.38,107.62,116.56,116.65,117.61$, $118.69,118.96,121.43,122.63,123.41,124.48,125.66,127.42,128.66,128.73,130.81,141.38$, $141.45,146.11,146.46,152.20,154.24,155.24,181.65,186.18$. Anal. Calcd. for $\mathrm{C}_{31} \mathrm{H}_{24} \mathrm{Cl}_{2} \mathrm{~N}_{2} \mathrm{O}_{3}$ : C, $68.51 \% ; \mathrm{H}, 4.45 \% ; \mathrm{N}, 5.15 \%$. Found: $\mathrm{C}, 68.86 ; \mathrm{H}, 4.70 \%$;, $5.42 \%$.

\section{References}

1. Josemin. (1998). Studies on reactions of Carbonyl compounds with Chloromethyleneiminium salts and Heterocumulenes. Ph.D. Thesis, Mahatma Gandhi University, Kottayam, India .

2. Jones, G.; Stanford, S. P. (2000). The Vilsmeier reaction of non-aromatic compounds. Org. React., $56,355-659$.

3. Jones, G.; Stanford, S. P. (1997). The Vilsmeier Reaction of Fully Conjugated Carbocycles and Heterocycles. Org. React., 49, 1.

4. Newman, M. S.; Sujeeth, P. K. (1978). Conversion of aromatic and .alpha.,.beta.-unsaturated aldehydes to dichlorides by thionyl chloride and dimethylformamide. J. Org. Chem., 43, 43674369.

5. Amaresh. A. A.; Perumal P. P. (1998). A novel one-pot synthesis of 2-aminoquinolines from arylazidoketones by cyclization under Vilsmeier conditions. Tetrahedron, 54, 14327-14340.

6. Zhao, L.; Liang, F.; Bie, X.;Sun, S. and Liu, Q. (2006). Efficient Synthesis of Highly Functionalized Dihydropyrido[2,3-d]pyrimidines by a Double Annulation Strategy from $\alpha$ Alkenoyl- $\alpha$-carbamoyl Ketene-(S,S)-acetals. J. Org. Chem., 71, 1094-1098.

7. Reddy, M. P.; Rao, G. S. K. (1981). Applications of the Vilsmeier reaction. 13. Vilsmeier approach to polycyclic aromatic hydrocarbons. J. Org. Chem., 46, 5371-5373.

8. Church, R.; Trust, R.; Albright, J. D.; Powell, D. W. (1995). New Synthetic Routes to 3-, 5-, and 6Aryl-2-chloropyridines. J. Org. Chem., 60, 3750-3758.

9. Mittelbach, M.; Junek, H. Syntheses with nitriles. (1982). 3,5-Dicyanopyridine derivatives by vilsmeier formylation of malononitrile and tetracyanopropenides. J. Heterocycl. Chem., 19, 1021-1024.

10. Amaresh, R. R.; Perumal, P. T. (1995). Ring opening of chalcone epoxides with vilsmeier reagent. Tetrahedron Lett., 36, 7287-7288.

11. Anderson, D. J. (1986). A reinvestigation of the Vilsmeier reaction of 3-phenyl-5-isoxazolinone. Isolation of 1,3-oxazin-6-ones. J. Org. Chem., 51, 945-947.

12. Fritz, H. (1959). Über $\alpha . \beta$-ungesättigte $\beta$-Anilino-carbonyl-Verbindungen als Mode- llsubstanzen für den Chromophor von C-Curarin-III (C-Fluorocurarin) Chem. Ber., 92, 1809-1817.

13. Baradarani, M. M.; Afghan, A.; Zebarjadi F.; Hasanzadeh K. and Joule , J. A. (2006). The synthesis of 3,3-dimethyl-2-(1-aryl-1H-pyrazol-4-yl)-3H-indoles. J. Heterocyclic Chem., 43, 1591-1595.

14. Rashidi A.; Baradarani, M. M. and Joule, J. A. (2011). Synthesis of 2,2'-bis(1-(2-aryl)-1Hpyrazol-4-yl)-3,3,3',3'-tetramethyl-3H,3'H-5,5'-biindoles and 2,7-bis(1-(3-aryl)-1H-pyrazol-4-yl)1,1,6,6-tetramethyl-1,6-dihydroindolo[7,6-g]indoles. Arkivoc, (ii), 252-259.

15. Rashidi A.; Afghan, A.; Baradarani M. M.; Joule, J. A. (2009). The Synthesis of 4-(3,3Dimethyl-3H-pyrrolo[2,3-f]quinolin-2-yl)pyrazoles and 4-(3,3-Dimethyl-3H-pyrrolo[3,2-h] quinolin-2-yl)pyrazoles. J. Heterocyclic Chem., 46, 428-431.

16. Simon, C.; Constantieux, T. and Rodriguez, J. (2004) Utilisation of 1,3-Dicarbonyl Derivatives in Multicomponent Reactions Eur. J. Org. Chem., 4957-4980.

17. Penning, T. D.; Talley, J. J.; Bertenshaw, S. R. et al. (1997). Synthesis and Biological Evaluation of the 1.5 Diarylpyrazole Class of Cyclooxygenase-2 Inhibitors: Identification of 4-[5-(4Methylphenyl)-3-(trifluoromethyl)-1H-pyrazole-1-yl]benzenesulfonamide(SC-58634, Celecoxib). J. Med. Chem. 40 (9): 1347-1365. 
18. Helliwell, M.; Baradarani, M. M.; Alyari, M.; Afghan, A. and Joule J. A. (2012). 2-(4-Chloro3,3,7-trimethyl-2,3-dihydro-1H-indol-2-ylidene)-2-cyanoacetamide ActaCrystallo- graphica Section E, E68, o234. 